\title{
Die Rekonstruktion früherer Stofffrachten in einem See mittels Sedimentuntersuchungen
}

\author{
Von D.M. Imboden, J. Tschopp und W. Stumm \\ Eidgenössische Technische Hochschule, EAWAG, Dübendorf
}

Manuskript eingegangen am 18.Juni 1979

\begin{abstract}
A mathematical model is presented describing the vertical concentration gradient of a chemical species in the sediments of a lake as a function of external input variation and of characteristic parameters (mass sedimentation rate or sinking velocity, distribution factor between dissolved and particulate phase, hydraulic loading). Delayed response of external input variations on sedimentation due to finite species residence time is taken into account. The model is tested for $\mathrm{Pb}, \mathrm{Cd}, \mathrm{Cu}$, and $\mathrm{Zn}$ in Greifensee (Switzerland) where - based on sediment data - an increase of external input of these metals by a factor $2.5(\mathrm{~Pb}, \mathrm{Cd}, \mathrm{Cu})$ and $4(\mathrm{Zn})$ is found for the last 35 years.
\end{abstract}

\section{Einleitung}

Natürliche Seen werden durch eine Vielzahl von anthropogen bedingten Mechanismen beeinflusst [1], wobei die Erhöhung der Zufuhr verschiedener Stoffe als eine der wichtigsten Störungen zu betrachten ist. Da für viele, heute als wichtige Verschmutzungsindikatoren betrachtete chemische Parameter historische Daten fehlen, ist es nicht immer möglich, die festgestellten Verhältnisse in Relation zu einem früheren, ungestörten Zustand zu setzen. Dies gilt insbesondere für die Schwermetalle, deren Zufuhr in manche Seen in jüngster Zeit deutlich zugenommen hat [2-4].

Als Folge von Sedimentations- und chemischen Austauschprozessen besteht ein direkter Zusammenhang zwischen dem Gewässerzustand eines Sees und der Zusammensetzung seiner Sedimente. Die chemische Charakteristik einer Sedimentschicht bleibt später, wenn die Dicke der Sedimente wächst und die Sedimentoberfläche stetig von der betreffenden Schicht wegwandert, mehr oder weniger stark erhalten. Allerdings können diagenetische Prozesse schon in den obersten Schichten zu chemischen Veränderungen führen $[5,6]$. Im Porenwasser gelöste Komponenten wandern infolge molekularer Diffusion oder Advektion [7]. Schliesslich können Organismen in den obersten Schichten partikuläre und gelöste Substanzen transportieren und damit zu einer vertikalen Durchmischung auch der partikulären Phase führen [8]. 
Immerhin deutet das Vorhandensein einer Schichtung (seien dies Jahresschichten oder andere Strukturen) darauf hin, dass in gewissen Seen die (mechanisch-)biologische Durchmischung fehlt oder höchstens von untergeordneter Bedeutung ist. Für Substanzen, welche hauptsächlich in der festen Phase des Sediments vorkommen und nicht direkt an den frühen diagenetischen Prozessen beteiligt sind, stellt demnach die vertikale Verteilung innerhalb der Sedimentkolonne ein gutes Bild dar über den zuräckliegenden zeitlichen Verlauf des Inputs dieser Substanz in das damals frische Sediment. Diese Feststellung gilt besonders für die im Sediment immobilen Schwermetalle [9].

Ist die Sedimentationsrate bekannt, kann aus den vertikalen Konzentrationsverläufen im Sediment im Prinzip die frühere Zufuhr des betreffenden Stoffes berechnet werden. Allerdings ist unter Umständen der direkte Zusammenhang zwischen externer Stoffzufuhr und Gehalt in der entsprechenden Jahresschicht kompliziert. Neben der erwähnten möglichen Wanderung des Stoffes im Sediment (Diffusion, Bioturbation) können zwei Faktoren eine Rolle spielen, nämlich erstens das Verhältnis zwischen zugeführter und im Sediment eingelagerter Stoffmenge (Seerückhalt) und zweitens die Zeitverzögerung zwischen externer Zufuhr und Sedimentation, welche sich als Folge der endlichen Aufenthaltszeit des Stoffes im See ergibt.

Das Ziel dieser Arbeit besteht in der Beantwortung der Frage, wie aus Messungen im Sediment auf den zeitlichen Verlauf der Zufuhr dieser Substanz in den See geschlossen werden kann und von welchen Faktoren diese Zusammenhänge bestimmt werden.

\section{Der See als vollständig durchmischtes System}

$\mathrm{Da}$ die Seen in unserer Klimazone im Jahr mindestens einmal tiefgreifend durchmischt werden, viele Seen sogar bis zur tiefsten Stelle, kann man über längere Zeiträume hinweg den See als vollständig durchmischtes System betrachten. Kurzzeitig (d.h. über Wochen oder einige Monate) stimmt diese Annahme bekanntlich nicht, vor allem nicht für jene Stoffe, welche an biologischen Vorgängen beteiligt sind.

Das in Abb. 1 dargestellte 1-Box-Modell führt für eine bestimmte Substanz (beschrieben durch die totale Konzentration $C_{\downarrow}$ ) zu folgender Bilanzgleichung:

$$
\mathrm{V} \frac{\mathrm{dC}}{\mathrm{dt}}=\mathrm{F}_{1}+\mathrm{F}_{3}-\mathrm{F}_{2}-\mathrm{F}_{4}
$$

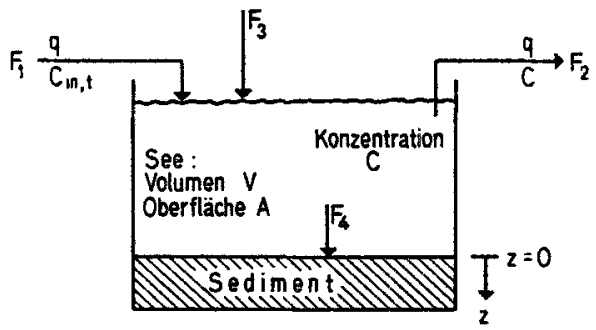

Abb. 1. 1-Box-Modell für die Beschreibung von Schwermetallen in einem See. Definition der Symbole vgl. Tabelle I.

Figure 1. l-box model for the balance of heavy metals in a lake. See table 1 for definition of symbols. 
Die Symbole sind in Tabelle 1 definiert; sie stimmen mit der Notation von Imboden und Lerman [10] überein. Wir werden im folgenden speziell den Fall von Spurenelementen betrachten, doch gelten die Überlegungen im Prinzip auch für andere Stoffe. Die beiden Inputfrachten $F_{1}$ und $F_{3}$ kann man durch die Ausdrücke

$$
\mathrm{F}_{1}=\mathrm{qC}_{\mathrm{t}, \mathrm{in}} ; \quad \mathrm{F}_{3}=\mathrm{AN}
$$

ersetzen.

Die Fracht im Abfluss $\left(\mathrm{F}_{2}\right)$ ist durch die totale Konzentration im See bestimmt:

$$
\mathrm{F}_{2}=\mathrm{q} \mathrm{C}_{\mathrm{t}} \text {. }
$$

Das Wasservolumen des Sees wird als konstant vorausgesetzt. Ferner sollen sich die Niederschläge auf die Seeoberfläche und die Verdunstungsrate kompensieren, so dass sich die Wasserbilanz auf die einfache Relation $\mathrm{q}_{\text {in }}=\mathrm{q}_{\mathrm{aus}}=\mathrm{q}$ reduziert.

Die totale Konzentration $C_{t}$ setzt sich aus der gelösten und der partikulären Fraktion zusammen.

$$
\mathrm{C}_{\mathrm{t}}=\mathrm{C}+\mathrm{C}_{\mathrm{g}} \mathrm{C}_{\mathrm{p}}
$$

Tabelle 1. Definition der verwendeten Bezeichnungen (analog zu [10]).

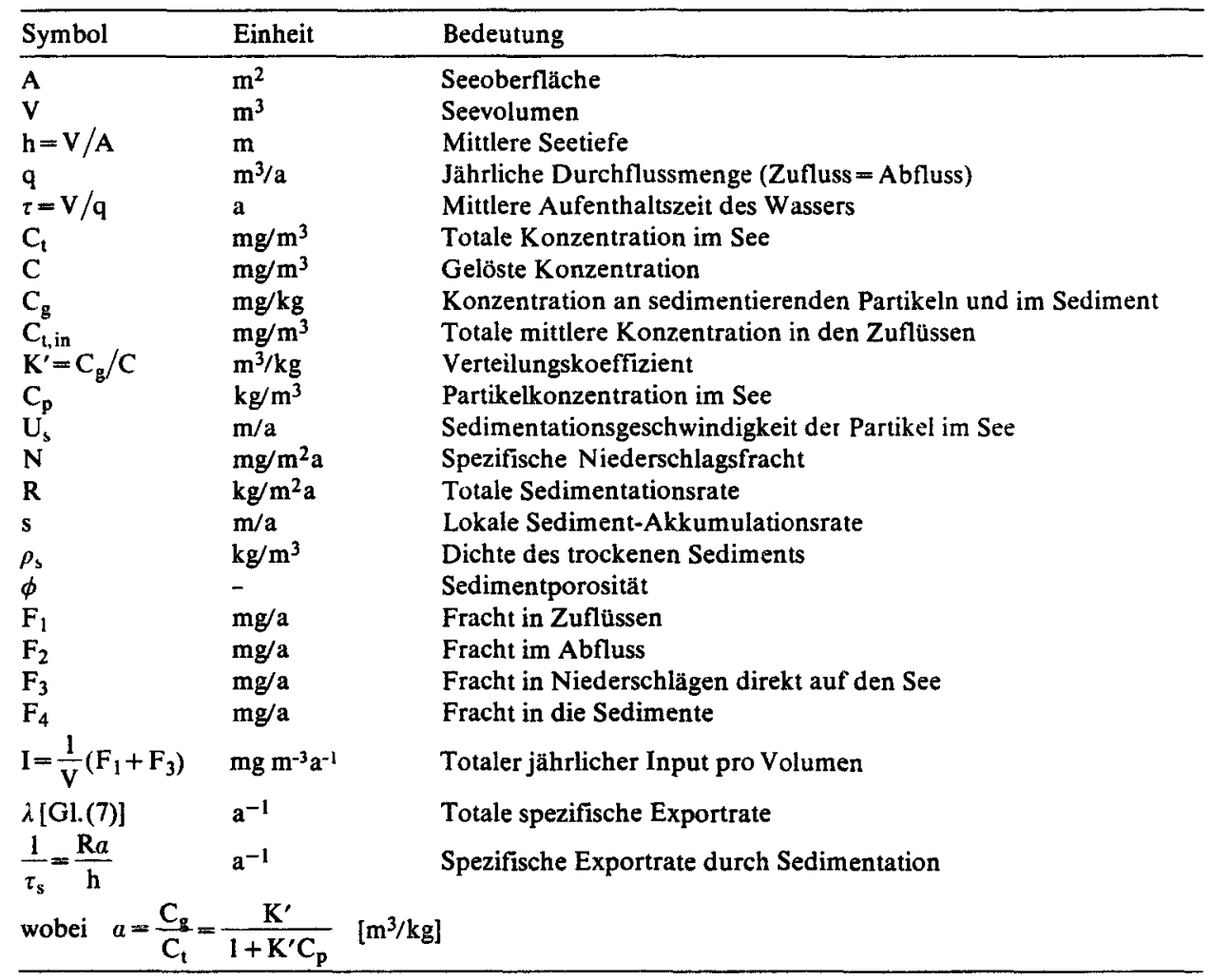


Table 1. Definition of variables (as in [10]).

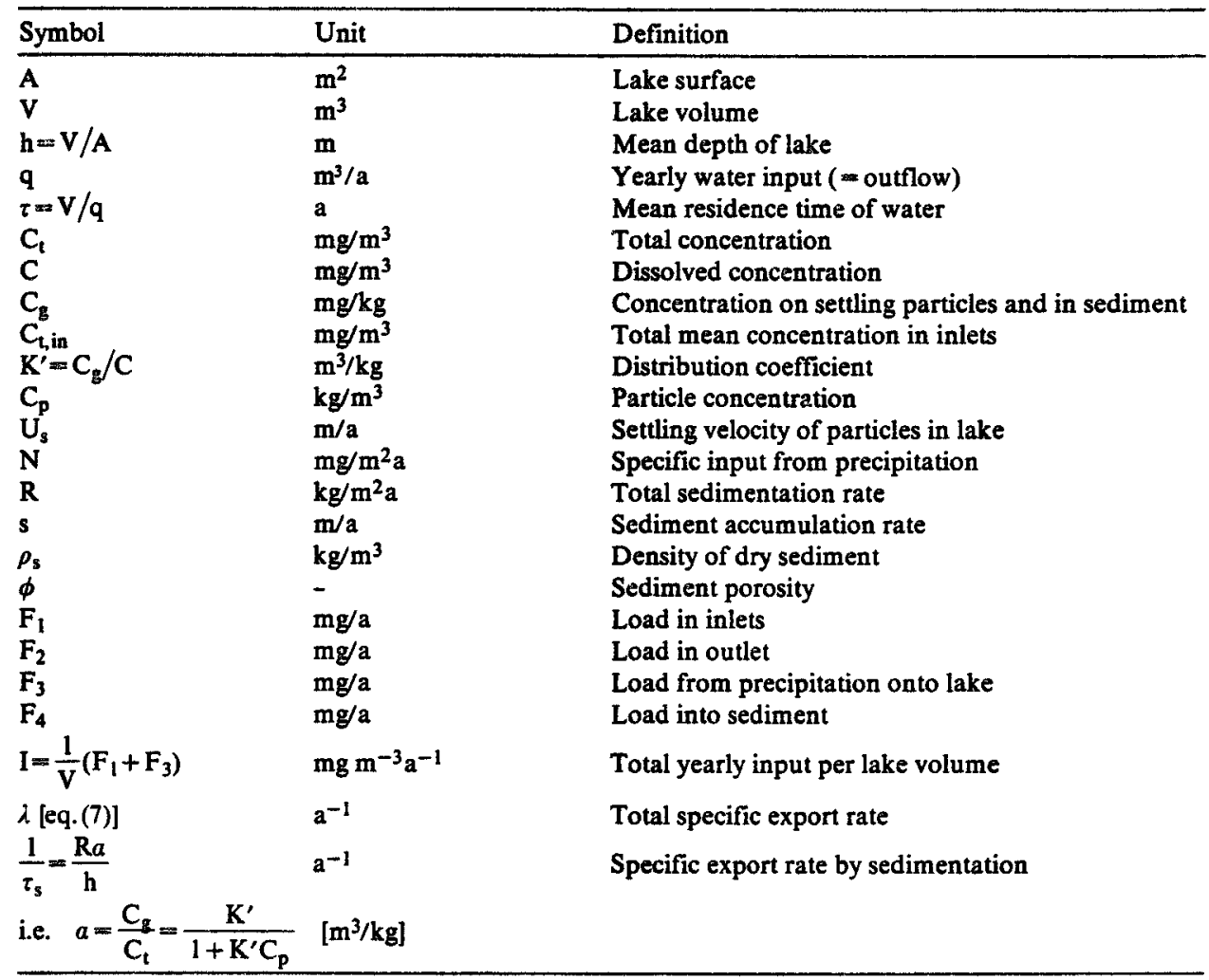

wobei zwischen dem gelösten Anteil $\mathrm{C}$ und der adsorbierten Konzentration pro Partikelmasse $\mathrm{C}_{\mathrm{g}}$ die Beziehung

$$
\mathrm{C}_{\mathrm{g}}=\mathrm{K}^{\prime} \mathrm{C}
$$

gelten soll. (Der Verteilungskoeffizient $K^{\prime}$ wird bei Stumm und Baccini [1] mit D bezeichnet.) Das Gleichgewicht (5) stellt sich unendlich rasch ein [11]. Aus Gl.(4) und (5) folgt

$$
\mathrm{C}_{\mathrm{g}}=a \mathrm{C}_{\mathrm{t}} \quad \text { mit } \quad a=\frac{\mathrm{K}^{\prime}}{1+\mathrm{K}^{\prime} \mathrm{C}_{\mathrm{p}}}
$$

Der Flux von Spurenelementen in die Sedimente $\mathrm{F}_{4}$ ist somit

$$
\mathrm{F}_{4}=\mathrm{ARC}_{\mathrm{g}}=a \mathrm{ARC} \text {. }
$$

Setzt man die Gl.(2), (3) und (7) in Gl.(1) ein, ergibt sich folgende lineare Differentialgleichung:

$$
\frac{\mathrm{dC}_{\mathrm{t}}}{\mathrm{dt}}=-\lambda \mathrm{C}_{\mathrm{t}}+\mathrm{I}
$$


mit

$$
\begin{aligned}
& \lambda=\frac{1}{\tau}+\frac{1}{\tau_{\mathrm{s}}}, \\
& \mathrm{I}=\frac{1}{\mathrm{~V}}\left(\mathrm{~F}_{1}+\mathrm{F}_{3}\right)=\frac{\mathrm{C}_{\mathrm{t}, \text { in }}}{\tau}+\frac{\mathrm{N}}{\mathrm{h}} .
\end{aligned}
$$

Die totale spezifische Exportrate $\lambda$ setzt sich aus zwei inversen Aufenthaltszeiten zusammen, aus derjenigen des Wassers

$$
\frac{1}{\tau}=\frac{q}{V}
$$

und aus derjenigen bezüglich Sedimentation

$$
\frac{1}{\tau_{\mathrm{s}}}=a \frac{\mathrm{RA}}{\mathrm{V}}=a \frac{\mathrm{R}}{\mathrm{h}}=\frac{\mathrm{K}^{\prime}}{1+\mathrm{K}^{\prime} \mathrm{C}_{\mathrm{p}}} \cdot \frac{\mathrm{R}}{\mathrm{h}} .
$$

Im allgemeinen sind $\lambda$ und I zeitabhängig, allerdings in unterschiedlicher Art. Der Koeffizient $i$ hängt von (möglicherweise zeitlich variablen) charakteristischen Seeparametern $\left(\tau, C_{p}, R\right)$ und nur über den Verteilungskoeffizienten $K^{\prime}$ vom betrachteten Metall ab. Umgekehrt ist die Inputgrösse I unabhängig von seeinternen Vorgängen; sie entspricht dem totalen jährlichen Metallinput pro Seevolumen. Diese Grösse soll aus den heute im Sediment gemessenen Konzentrationen bestimmt werden.

Die allgemeinste Lösung der linearen Differentialgleichung (8) mit zeitabhängigen Koeffizienten findet sich in jedem Lehrbuch über gewöhnliche Differentialgleichungen. Sie lautet mit der Anfangsbedingung $C_{t}(t=0)=C_{t}^{0}$ :

$$
C_{t}(t)=C_{t}^{0} e^{-\omega(t)}+e^{-\omega(t)} \int_{0}^{t} e^{\omega\left(t^{\prime}\right)} I\left(t^{\prime}\right) d t^{\prime} \text { mit } \omega(t)=\int_{0}^{t} \lambda\left(t^{\prime}\right) d t^{\prime}
$$

Für zeitlich konstantes $\lambda$ und I folgt

$$
C_{t}(t)=\frac{I}{\lambda}+\left(C_{t}^{0}-\frac{I}{\lambda}\right) e^{-i t}
$$

Dieser Ausdruck strebt nach einer Zeit $t \gg 1 / \lambda$ gegen die stationäre Konzentration

$$
\mathrm{C}_{\mathrm{t}}(\infty)=\frac{\mathrm{I}}{\lambda}
$$

Die lokale Sediment-Akkumulationsrate $s$ in der Tiefe $z_{0}$ hängt mit der Sedimentationsrate $\mathrm{R}$ zur Zeit $\mathrm{t}_{0}$ (Zeit, als die Schicht bei $\mathrm{z}_{0}$ frisch sedimentierte) durch die Beziehung 


$$
\mathrm{R}=\mathrm{s} \rho_{\mathrm{s}}(1-\phi)
$$

zusammen. Falls die Sedimentation als ein Prozess 1. Ordnung beschrieben werden kann, so ist $R=U_{s} C_{p}$, wobei $U_{s}$ die mittlere Sinkgeschwindigkeit der Partikel bedeutet. Es folgt

$$
\mathrm{C}_{\mathrm{p}}=\frac{\mathrm{R}}{\mathrm{U}_{\mathrm{s}}}=\frac{s \rho_{\mathrm{s}}(1-\phi)}{\mathrm{U}_{\mathrm{s}}} .
$$

Durch Einsetzen von Gl.(17) in Gl.(12) kann man die relative Aufenthaltszeit der betrachteten Substanz bezüglich Sedimentation durch die Sedimentationsrate $R$ ausdrücken:

$$
\tau_{\mathrm{s}}=\left(\frac{1}{\mathrm{~K}^{\prime} \mathrm{R}}+\frac{1}{\mathrm{U}_{\mathrm{s}}}\right) \mathrm{h} .
$$

\section{Zusammenhang zwischen Metallkonzentration im Sediment und früherer Metallbelastung}

Die Metallkonzentration pro Trockensubstanz im frischen Sediment ist gleich der Konzentration an sedimentierenden Partikeln $\left(C_{\mathrm{g}}\right)$ und kann somit über Gl.(6) als Funktion der totalen Konzentration ausgedrückt werden. Die zeitliche Veränderung von $\mathrm{C}_{\mathrm{g}}$ ist somit nach $\mathrm{Gl} .(8)$

$$
\frac{\mathrm{dC}_{\mathrm{g}}}{\mathrm{dt}}=\frac{\mathrm{d}}{\mathrm{dt}}\left(\alpha \mathrm{C}_{\mathrm{V}}\right)=-\lambda \mathrm{C}_{\mathrm{g}}+a \mathrm{I}+\mathrm{C}_{\mathrm{g}}\left(\frac{1}{a} \cdot \frac{\mathrm{d} a}{\mathrm{dt}}\right)
$$

Anderseits hängt $\mathrm{dC}_{\mathrm{g}} / \mathrm{dt}$ mit der heute im Sediment vorgefundenen vertikalen Konzentrationsverteilung in einfacher Art zusammen, falls für das betreffende Spurenmetall vertikale Transportvorgänge im Sediment vernachlässigt werden können:

$$
\frac{d C_{g}}{d t}=\frac{d C_{g}}{d z} \cdot \frac{d z}{d t}=-s \frac{d C_{g}}{d z}
$$

z: Tiefe im Sediment, gemessen von der Sedimentoberfläche. Einsetzen von Gl. (20) in Gl.(19) und Auflösen nach I liefert

$$
\mathrm{I}\left(\mathrm{t}_{0}\right)=\frac{1}{a}\left[-\left.\mathrm{s}\left(\mathrm{z}_{0}\right) \frac{\mathrm{dC} \mathrm{C}_{\mathrm{g}}}{\mathrm{dz}}\right|_{\mathrm{z}_{0}}+\dot{\lambda}\left(\mathrm{z}_{0}\right) \mathrm{C}_{\mathrm{g}}\left(\mathrm{z}_{0}\right)-\left(\frac{1}{a} \cdot \frac{\mathrm{d} a}{\mathrm{dt}}\right) \mathrm{C}_{\mathrm{g}}\left(\mathrm{z}_{0}\right)\right] .
$$

Nimmt man vorerst an, der Verteilungskoeffizient $\mathrm{K}^{\prime}$ und die Partikelkonzentration im See $C_{p}, d . h$. also $a$, seien auch unter dem Einfluss eines variablen Inputs I konstant, so kann der (zurückliegende) Input I zur Zeit $t_{0}$ aus einem Ausdruck berechnet werden, der sich allein aus Sedimentdaten der Schicht in der Tiefe $z_{0}$ er- 
gibt. Man beachte, dass Gl.(21) - abgesehen von der Annahme, es treten nachträglich im Sediment keine Transportphänomene auf - völlig allgemein ist und beispielsweise nicht von den s- bzw. $\lambda$-Werten zu einem späteren Zeitpunkt abhängt. Natürlich muss der Zusammenhang zwischen $t_{0}$ und $z_{0}$ bekannt sein, was beim Fehlen unabhängiger Altersbestimmungen auf eine Berechnung der Sedimentation zwischen $t_{0}$ und der Gegenwart $t$ hinausläuft. Für konstantes $s$ ist

$$
\mathrm{z}_{0}=\mathrm{s}\left(\mathrm{t}-\mathrm{t}_{0}\right) \text {. }
$$

Wir wollen im folgenden die Bedeutung von Gl.(21) durch die Diskussion einiger Spezialfälle veranschaulichen und dabei weiterhin $a=$ konstant voraussetzen:

1. Der Input I ist zeitlich konstant, die Konzentration im Sediment ist somit stationär, d.h. $\mathrm{dC}_{\mathrm{g}} / \mathrm{dz}=0$. Dann folgt

$$
\mathrm{I}=\frac{\lambda}{a} \mathrm{C}_{\mathrm{g}}=\lambda \mathrm{C}_{\mathbf{t}}
$$

Diese Gleichung ist identisch mit der stationären Lösung [Gl.(15)].

2. $\left.\frac{d C_{g}}{d z}\right|_{z_{0}}>0: C_{g}$ nimmt gegen die jüngeren Sedimentschichten hin ab. Nach Gl.(21) ist somit $\mathrm{I}<(i / a) \mathrm{C}_{\mathrm{g}}$. Dies bedeutet, dass der Metallgehalt im See von einem früheren, höheren Input her noch grösser ist als der zum momentanen Input gehörende stationäre Wert.

3. $\left.\frac{\mathrm{dC}_{\mathrm{g}}}{\mathrm{dz}}\right|_{\mathrm{z}_{0}}<0: \mathrm{C}_{\mathrm{g}}$ nimmt gegen die jüngeren Sedimentschichten hin zu. Es ist $\mathrm{I}>(\lambda / a) \mathrm{C}_{\mathrm{g}}$. Dieser Fall tritt ein, wenn die Belastung I zeitlich zunimmt, die Konzentration im See bzw. im Sediment aber infolge der nur endlich grossen Responsezeit des Systems mit einer gewissen zeitlichen Verzögerung wächst.

Der Prozess der Sedimentation sowie das hier verwendete 1-Box-Modell schliessen eine zeitliche Auflösung der Metallkonzentrationen, welche feiner ist als etwa ein Jahr, von vornherein aus. Nach Gl.(14) darf daher bis auf Korrekturterme von wenigen Prozenten angenommen werden, für $i>2 \mathrm{a}^{-1}$ befänden sich Zufuhr und Konzentration im See auch bei variierendem Input ständig im Gleichgewicht [Gl. (15)]. Das bedeutet auch. dass in Gl.(21) der Term mit den Konzentrationsgradienten, $\mathrm{dC}_{\mathrm{g}} / \mathrm{dz}$. vernachlässigt werden kann.

Tatsächlich liegen die typischen Aufenthaltszeiten für Seen zwischen 1 und 100 Jahren, so dass in Gl. (9) der erste Term $(1 / \tau)$ allein kaum $\lambda>2 \mathrm{a}^{-1}$ garantiert. Auf der andern Seite sind die Verteilungskoeffizienten $\mathrm{K}^{\prime}$ gerade für jene Substanzen gross, welche im Sediment relativ immobil sind (z. B. Schwermetalle). In diesen Fällen ist - ausser im Fall einer extrem niedrigen Akkumulationsrate s oder/und einer sehr grossen mittleren Seetiefe $\mathrm{h}$ - nicht nur $i>2 \mathrm{a}^{-1}$ erfüllt, sondern zugleich auch $1 / \tau \ll 1 / \tau_{s}$, so dass

$$
\lambda \sim \frac{\mathrm{I}}{\tau_{\mathrm{s}}}=a \frac{\mathrm{R}}{\mathrm{h}}
$$


folgt und nach Gl.(21) die Zufuhr von Metallen in die Sedimente unabhängig von $a, d$.h. unabhängig vom Verteilungskoeffizient $\mathrm{K}^{\prime}$, wird:

$$
\mathrm{I}=\frac{\hat{\lambda}}{a} \mathrm{C}_{\mathrm{g}}=\frac{\mathrm{R}}{\mathrm{h}} \mathrm{C}_{\mathrm{g}}=\frac{\mathrm{s}}{\mathrm{h}} p_{\mathrm{s}}(1-\phi) \mathrm{C}_{\mathrm{g}} \text {. }
$$

Der Grund liegt darin, dass in diesem Fall die Substanz den See praktisch nur via Sedimentation verlässt, der Input sich also vollständig in der zur entsprechenden Zeitperiode gehörenden Sedimentschicht wiederfindet. Der Zusammenhang zwischen $\mathrm{I}$ und $\mathrm{C}_{\mathrm{g}}$ reduziert sich auf eine triviale Stoffbilanz.

\section{Anwendung: Spurenmetalle im Greifensee}

In Abb. 2 sind von Tschopp [4] gemessene durchschnittliche Schwermetallgehalte im Greifensee dargestellt. Die Sedimentstiche wurden in den Jahren 1975 und 1976 gezogen. Die Sedimentationsraten sind von Gäggeler et al. [12] anhand der ${ }^{210} \mathrm{~Pb}-$ Methode bestimmt worden. Resultate lassen vermuten, dass die $\mathrm{Zufuhr}$ an $\mathrm{Cu}, \mathrm{Zn}$, $\mathrm{Cd}$ und $\mathrm{Pb}$ in den See in den vergangenen 50 Jahren zugenommen hat. Im Falle von $\mathrm{Cu}, \mathrm{Zn}$ und $\mathrm{Cd}$ nimmt die Konzentration in den obersten $5 \mathrm{~cm}$ (entspricht der Sedimentation während der letzten 6 bis 7 Jahre) wieder ab. Eine Interpretation
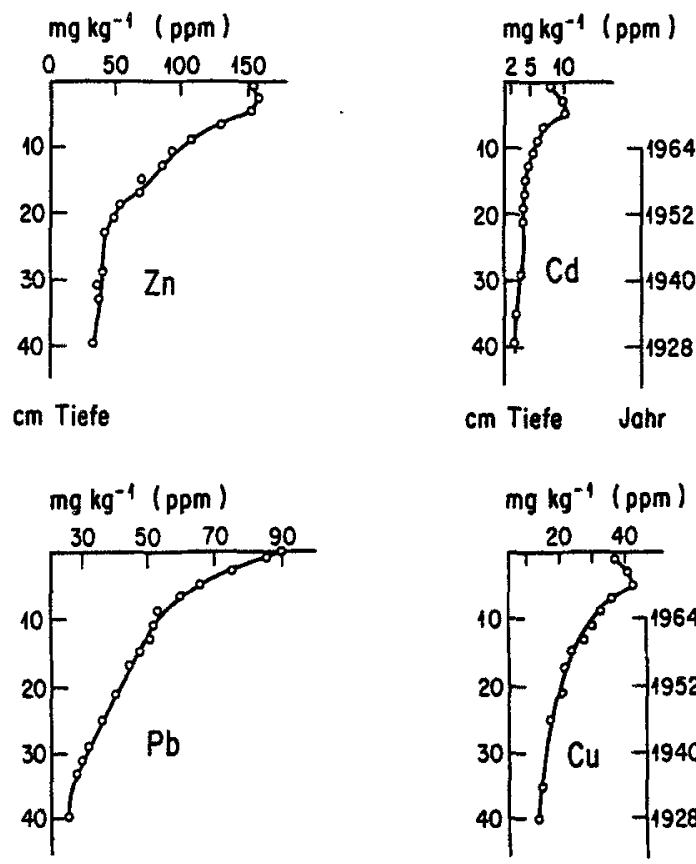

cm Tiefe

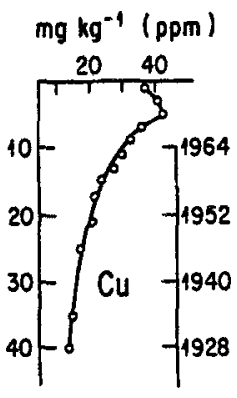

cm Tiefe Jahr

Abb.2. Durchschnittliche Schwermetallgehalte in Sedimentprofilen von sieben Probenahmestellen im Greifensee, nach Tschopp [4].

Figure 2. Mean heavy metal concentration in sediment cores from seven sampling sites in Greifensee, from Tschopp [4]. 
dieses Sachverhaltes ist schwierig: Da nach Emerson [6] die Sedimentationsrate im Greifensee, ausgedrückt als Trockensubstanz pro Fläche und Zeit, in den letzten Jahren ziemlich konstant war, könnte es sich tatsächlich um eine echte Reduktion des Metallinputs in die Sedimente und damit um eine Verkleinerung der externen Belastung handeln. Anderseits ist das Sediment in den obersten Schichten noch recht instabil und beinahe flüssig, so dass eine quantitative Probenahme schwierig ist. Auch nimmt in den obersten $4 \mathrm{~cm}$ der Gehalt an organischem Material von $20 \%$ auf etwa $12 \%$ ab, was bei konstantem absolutem Metallgehalt einen Anstieg des Gehaltes pro Trockengewicht um $8 \%$ zur Folge hätte. Erst ein Anhalten dieses abfallenden Trends könnte die Hypothese einer Inputreduktion erhärten. Aus diesen Gründen werden wir im folgenden nur die Sedimentschichten unterhalb $5 \mathrm{~cm}$ betrachten, wo die Konzentration mit zunehmender Tiefe stetig abnimmt. Die heutige Entwicklung der Metallzufuhr in den Greifensee müsste durch weitere Sedimentuntersuchungen abgeklärt werden. In Tabelle 2 sind die wichtigsten Daten über den Greifensee zusammengestellt und die in Gl. (16) bis (18) behandelten Parameter berechnet, welche das Verhalten der Partikel und der Metalle im See charakterisieren.

Tschopp [4] hat für die betreffenden Schwermetalle Verteilungskoeffizienten $\mathrm{K}^{\prime}$ bestimmt, welche mit andern Werten [11] ziemlich gut übereinstimmen. In Tabelle 3 sind die entsprechenden Aufenthaltszeiten bezüglich Sedimentation $\left(\tau_{\mathrm{s}}\right)$ und die totale spezifische Exportrate $\lambda$ berechnet. Anhand der gemessenen Metallkonzentration im Sediment in Abhängigkeit der Tiefe (Abb.2) können die in Gl.(21) auftretenden Terme abgeschätzt werden. Tatsächlich ergibt sich in allen Fällen die Beziehung

$$
\mathrm{s} \frac{\mathrm{dC} \mathrm{g}}{\mathrm{dz}} \ll \lambda \mathrm{C}_{\mathrm{g}}
$$

Tabelle 2. Allgemeine Daten über den Greifensee.

Table 2. Characteristic data on Greifensee (definitions see table 1).

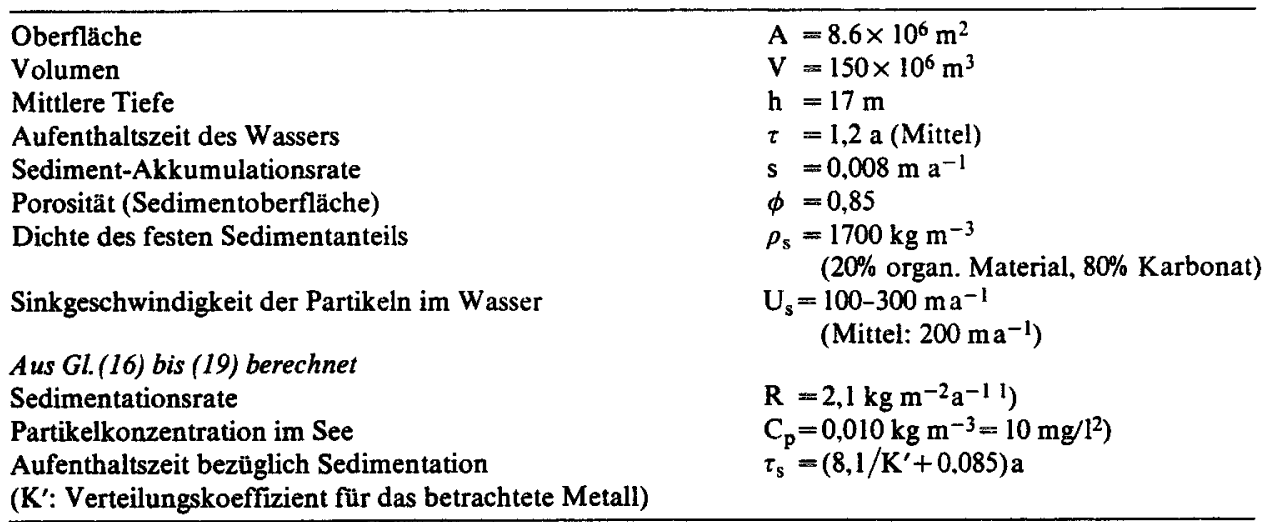

1) Emerson und Widmer [6] berechneten $\mathrm{R} \mathrm{u}(1,5 \pm 0,5) \mathrm{kg} \mathrm{m}^{-2} \mathrm{a}^{-1}$; doch sind ihre aus Warven bestimmten Sedimentationsgeschwindigkeiten $s$ kleiner als die auf der $210 \mathrm{~Pb}-\mathrm{Messung}$ basierenden Werte.

2) Messungen $15 \pm 5 \mathrm{mg} / \mathrm{l}[4]$. 
Tabelle 3. Charakteristische Parameter für einige Spurenmetalle im Wasser und Sediment des Greifensees.

Table 3. Characteristic parameters for some trace metals in the water and sediments of Greifensee (Switzerland).

\begin{tabular}{|c|c|c|c|c|c|c|}
\hline & $\begin{array}{l}K^{\prime} \\
\text { nach [4] } \\
\left(\mathrm{m}^{3} / \mathrm{kg}\right)\end{array}$ & $\begin{array}{l}1 / \tau_{\mathrm{s}} \\
{[G L .(18)]} \\
\left(a^{-1}\right)\end{array}$ & $\begin{array}{l}i \\
{[G L(9)]} \\
\left(a^{-1}\right)\end{array}$ & $\begin{array}{l}\mathrm{C}_{\mathrm{g}}(5 \mathrm{~cm}) \\
\text { (Abb.2) } \\
(\mathrm{mg} / \mathrm{kg})\end{array}$ & $\begin{array}{l}\lambda \mathrm{C}_{\mathrm{g}} \\
(\mathrm{mg} \\
\left.\mathrm{kg}^{-1} \mathrm{a}^{-1}\right)\end{array}$ & 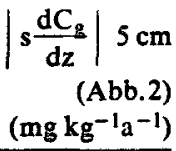 \\
\hline $\mathbf{P b}$ & 120 & 6,7 & 7,5 & 80 & 600 & 4,8 \\
\hline $\mathrm{Cd}$ & 65 & 4,8 & 5,6 & 10 & 56 & 1,1 \\
\hline $\mathrm{Cu}$ & 35 & 3,1 & 4,0 & 40 & 160 & 3,2 \\
\hline $\mathrm{Zn}$ & 25 & 2,4 & 3,3 & 150 & 495 & 6,0 \\
\hline
\end{tabular}

was bedeutet, dass sich der Greifensee bezüglich eines zeitlich variierenden Metallinputs immer im stationären Zustand befunden hat. Für die Sedimentschicht in $5 \mathrm{~cm}$ Tiefe kann daher mit einer vereinfachten Form von Gl.(21) die totale jährliche Inputrate in den Greifensee berechnet werden (Tab.4). Die entsprechenden Werte wären für die Sedimentoberfläche, d.h. für die Zeit der Probenahme $(1975 / 76)$, kleiner als diejenigen in Tabelle 4, falls die für $\mathrm{Cd}, \mathrm{Cu}$ und $\mathrm{Zn}$ beobachteten Konzentrationsabnahmen gegen die Oberfläche hin einer echten Abnahme der Metallzufuhr entsprächen (vgl. vorn). Der Vergleich mit der Berechnung des totalen Inputs aus Messungen von Niederschlägen und Zuflüssen für diese Zeit [4] unterstützt die Vermutung einer Reduktion der Zufuhr in jüngster Zeit - ausser für Blei, wo ja auch die Sedimente keinen Konzentrationsrückgang zeigen.

Im Falle des Cadmiums wird entweder die Konzentration im Sediment systematisch überschätzt, oder Cd gelangt hauptsächlich auf einem Weg in den See, der bei der Zufuhruntersuchung nicht erfasst worden ist. Für die erste Annahme spre-

Tabelle 4. Vergleich von aus Sedimentgehalten bestimmten Inputraten einiger Spurenmetalle in den Greifensee mit Werten, die aus Zufluss- und Niederschlagsmessungen berechnet sind.

Table 4. Comparison of input rates of trace metals calculated from sediment concentrations and from concentrations in inlets and precipitations, respectively.

\begin{tabular}{|c|c|c|c|c|}
\hline & $\begin{array}{l}\text { Für die Ze } \\
\text { Rückhalt }\end{array}$ & $\begin{array}{l}\left.1975 / 76^{1}\right) \\
\text { Input } \\
\text { berechnet } \\
{[\mathrm{Gl} .(21)]} \\
(\mathrm{kg} / \mathrm{a})\end{array}$ & $\begin{array}{l}\text { Messung } \\
\text { Zuflüsse/ } \\
\text { Niederschläge } \\
\text { (kg/a) }\end{array}$ & $\begin{array}{r}\text { Jahr } 1940 \\
\text { Input } \\
\text { berechnet } \\
\left.[\mathrm{Gl} .(21)]^{2}\right) \\
(\mathrm{kg} / \mathrm{a})\end{array}$ \\
\hline $\mathbf{P b}$ & 0,88 & 1640 & 1660 & 620 \\
\hline $\mathrm{Cd}$ & 0,85 & 215 & 15 & 6 \\
\hline $\mathrm{Cu}$ & 0,78 & 920 & 560 & 200 \\
\hline$Z_{\mathbf{n}}$ & 0,73 & 3700 & 3100 & 800 \\
\hline
\end{tabular}

1) Berechnet aus Metallgehalten in $5 \mathrm{~cm}$ Sedimenttiefe bzw. aus Untersuchungen an Zuflüssen und Niederschlägen für die Jahre 1975/76.

2) Berechnet aus Metallgehalten in $30 \mathrm{~cm}$ Tiefe unter Annahme eines zeitlich konstanten Verteilungskoeffizienten $K^{\prime}$, konstanter Partikelkonzentration $C_{p}$ und konstanter Sedimentationsrate $R$. Die letzte Annahme wird durch die Untersuchungen von Emerson und Widmer [6] gestïtzt. 
chen Untersuchungen an Bodensee-Sedimenten [2], wo bei ähnlichen Gehalten an $\mathrm{Pb}, \mathrm{Cu}$ und $\mathrm{Zn}$ nur 0,4 ppm $\mathrm{Cd}$ gemessen wurde. Gegen die zweite Annahme spricht die Feststellung von Baccini [3], wonach im Alpnachersee der atmosphärische Input pro Fläche von der gleichen Grössenordnung wie im Greifensee ist.

Der Seerückhalt, d.h. das Verhältnis zwischen zugeführter und ins Sediment eingelagerter Stoffmenge, ist im stationären Zustand durch das Verhältnis $\left(1 / \tau_{\mathrm{s}}\right) / \lambda$ gegeben. Gl.(23) und (24) entsprechen demnach einer Vereinfachung, bei welcher der Seerückhalt als $100 \%$ angenommen wird unter Vernachlässigung jenes Anteils, der den See via den Abfluss verlässt.

Falls $a$ konstant ist, besteht eine lineare Beziehung zwischen der Sedimentkonzentration $\mathrm{C}_{\mathrm{g}}$ und der Zufuhr während jener Zeit, in der die entsprechende Sedimentschicht abgelagert worden ist. Aus Abb. 2 kann in diesem Fall der frühere Verlauf der Metallzufuhr in den Greifensee leicht rekonstruiert werden. Berechnungen für das Jahr 1940 finden sich in Tabelle 4.

\section{Der Einfluss eines variablen Verteilungsgleichgewichtes}

Das in Gl.(6) beschriebene Verhältnis (a) zwischen totaler Metallkonzentration $\left(C_{f}\right)$ und der Konzentration pro Partikelmasse $\left(C_{g}\right)$ kann sich aus zwei Gründen zeitlich verändern:

1. Veränderung der Partikelkonzentration im See $C_{p}$ als Folge eines veränderten Inputs, einer veränderten In-situ-Produktion (Biomasse) oder veränderter Sedimentationsgeschwindigkeit $\mathrm{V}_{\mathrm{s}}$.

2. Veränderung des Verteilungskoeffizienten $K^{\prime}$ als Folge nichtlinearer Effekte bei veränderter externer Zufuhr oder wegen veränderter Eigenschaften (Oberfläche) der Partikel im See.

Der in Gl.(21) auftretende Term, welcher die zeitliche Variation von $\alpha$ enthält, ist:

$$
\frac{1}{a} \cdot \frac{\mathrm{d} a}{\mathrm{dt}}=\left(\frac{1}{\mathrm{~K}^{\prime}} \cdot \frac{\partial \mathrm{K}^{\prime}}{\partial \mathrm{t}}\right) \frac{1}{1+\mathrm{K}^{\prime} \mathrm{C}_{\mathrm{p}}}-\left(\frac{1}{\mathrm{C}_{\mathrm{p}}} \cdot \frac{\partial \mathrm{C}_{\mathrm{p}}}{\partial \mathrm{t}}\right) \frac{\mathrm{K}^{\prime} \mathrm{C}_{\mathrm{p}}}{1+\mathrm{K}^{\prime} \mathrm{C}_{\mathrm{p}}}
$$

Die bei den relativen Ableitungen stehenden Koeffizienten liegen zwischen 0 und 1 , so dass praktisch immer die Ungleichung $(\mathrm{a}=\mathrm{Jahr})$

$$
\frac{1}{a} \cdot \frac{\mathrm{d} a}{\mathrm{dt}} \ll 1 \mathrm{a}^{-1}
$$

gültig ist, es sei denn, $K^{\prime}$ oder $C_{p}$ änderten sich in einem einzigen Jahr um 100 oder mehr Prozent, was höchst unwahrscheinlich wäre. Da umgekehrt in den meisten Fällen $\lambda$ grösser als $1 \mathrm{a}^{-1}$ ist, kann in Gl.(21) der Term mit $\mathrm{d} a / \mathrm{dt}$ gegenüber dem zweiten Term $\left(\lambda C_{g}\right)$ vernachlässigt werden. Trotzdem stellt sich das Problem, in Gl. (21) das zu einem früheren Zeitpunkt $t_{0}$ gültige $a$ und daraus den früheren Input $\mathrm{zu}$ bestimmen. Allerdings wird für stark adsorbierende Substanzen $\lambda$ die Berechnung der Zufuhr unabhängig von $\alpha$ [Gl.(24)]. 


\section{Schlussfolgerungen}

1. Für Substanzen mit fehlender oder zumindest beschränkter Mobilität im Sediment kann aus Konzentrationsprofilen im Sediment auf den zurückliegenden zeitlichen Verlauf der Zufuhr dieses Stoffes in den See geschlossen werden. Die gefundene Beziehung [Gl.(21)] gilt auch für jene Fälle, wo eine rasche Veränderung bei der Zufuhr zu einer (vorübergehenden) Ungleichgewichtssituation zwischen Input und Konzentration im See führt, zumindest falls der Verteilungskoeffizient $K^{\prime}$ und die Partikelkonzentration $C_{p}$ nicht extreme zeitliche Schwankungen aufweisen.

2. Die totale spezifische Exportrate $\lambda$ setzt sich aus zwei Termen zusammen

$$
\lambda=\frac{1}{\tau}+\frac{1}{\tau_{\mathrm{s}}}
$$

welche den Export im Abfluss bzw. in die Sedimente beschreiben. Der Seerückhalt ist im stationären Zustand durch das Verhältnis $\left(1 / \tau_{\mathrm{s}}\right) / \lambda$ gegeben.

3. Für Substanzen mit grossem Verteilungskoeffizient $K^{\prime}$ und bei nicht zu kleinen Partikelkonzentrationen $\left(C_{p}>0,01 \mathrm{~kg} / \mathrm{m}^{3}\right)$ ist $\lambda$ sicher grösser als $2 \mathrm{a}^{-1}$. In diesem Fall kann der Term $\mathrm{sdC}_{\mathrm{g}} / \mathrm{dz}$ in Gl.(21), welcher ein mögliches Ungleichgewicht zwischen Input und Konzentration im See ausdruckt, vernachlässigt werden. Zudem sind die Sedimentgehalte $C_{g}$ und der Input I zueinander proportional, falls der Verteilungskoeffizient $K^{\prime}$ und die Partikelkonzentration im See $C_{p}$ zeitlich konstant sind. In diesem Fall gibt der relative Verlauf von $\mathrm{C}_{g}$ ein direktes Abbild des Verlaufes der früheren Stoffzufuhr in den See.

4. Für stark adsorbierende Stoffe (z.B. gewisse Schwermetalle) kann derjenige Anteil, welcher den See via Abfluss verlässt, gegenüber der Einlagerung ins Sediment vernachlässigt werden $\left(\lambda \sim 1 / \tau_{\mathrm{s}}\right)$. In diesem Fall gibt die Sedimentkonzentration $\mathrm{C}_{\mathrm{g}}$ ein direktes quantitatives Mass für die zurückliegende Zufuhr, welches unabhängig von Verteilungskoeffizient $K^{\prime}$ und Partikelkonzentration $C_{p}$ ist.

5. Das Beispiel der Spurenmetalle stellt wegen der kurzen Aufenthaltszeit im See einen Extremfall dar, für den offensichtlich der Zusammenhang zwischen Zufuhr und Sedimentgehalt einfach ist. Auf der andern Seite können Stoffe mit geringem Rückhalt (konservative Stoffe) sehr grosse Aufenthaltszeiten besitzen, dies natürlich vor allem in Seen mit grosser Füllzeit $\tau$. Für diese Fälle kann auch der nichtstationäre Term in Gl.(21) wichtig sein; ein einfacher Zusammenhang zwischen Sedimentgehalt und früherer Zufuhr besteht dann nicht mehr, wenn nicht auf die hier entwickelte Theorie, insbesondere auf Gl.(21), zurückgegriffen wird.

\section{Zusammenfassung}

Der Zusammenhang zwischen vertikaler Stoffverteilung im Sediment eines Sees und der zeitlichen Entwicklung der externen Stoffzufuhr wird durch vier Faktoren kompliziert: 1. Diagenetische Prozesse im Sediment, 2. vertikaler Stofftransport im Sediment (Diffusion im Porenwasser, Bioturbation), 3. zeitliche Veränderung des Seerückhaltes und 4. Zeitverzögerung zwischen externer Zufuhr und Sedimentation als Folge der endlichen Aufenthaltszeit des Stoffes im See. 
Für konservative Substanzen in Sedimenten, welche Schichtstrukturen aufweisen, spielen die ersten beiden Faktoren keine Rolle, falls sich die chemische Komponente vor allem in der festen Phase konzentriert (Beispiel Spurenmetalle). Für diesen Spezialfall wird ein mathematisches Modell entwickelt, welches für zeitlich variablen externen Input den vertikalen Konzentrationsverlauf im Sediment als Funktion gewisser charakteristischer Parameter beschreibt (Sedimentationsrate im See bzw. Partikelkonzentration und Sinkgeschwindigkeit, mittlere Seetiefe, hydraulische Belastung, Verteilungskoeffizient der Substanz zwischen adsorbierter und gelöster Phase). Das Modell wird im Greifensee für die Metalle $\mathrm{Pb}, \mathrm{Cd}$, $\mathrm{Cu}$ und $\mathrm{Zn}$ getestet. Danach hat die externe Zufuhr dieser Metalle von 1940 bis 1975 um den Faktor 2,5 ( $\mathrm{Pb}, \mathrm{Cd}, \mathrm{Cu})$ bis $4(\mathrm{Zn})$ zugenommen.

Als Folge des grossen Verteilungskoeffizienten liegt der Seerückhalt der Spurenmetalle zwischen $73 \%(\mathrm{Zn})$ und $88 \%(\mathrm{~Pb})$; die Sedimente befinden sich trotz variabler externer Belastung im Gleichgewicht mit der Belastung. Eine Abnahme der Metallgehalte in den obersten $5 \mathrm{~cm}(\mathrm{Cd}, \mathrm{Cu}, \mathrm{Zn})$ könnte auf einen jüngsten Belastungsrückgang hinweisen, doch müsste diese Hypothese durch spätere Messungen noch überprüft werden.

\section{SUMMARY}

The evaluation of former input rates into a lake from sediment analysis

The relationship between the temporal variation of external input and the vertical distribution of a chemical species in the sediment column of a lake is influenced by four factors: 1. Diagenetic processes in the sediment, 2 . vertical transport in the sediment column by diffusion or bioturbation, 3. temporal variation in the retention factor, and 4. delayed response of external input variations on sedimentation due to the finite species residence time in the system.

For sediments with layer structure and for conservative substances occurring mainly in the solid fraction, the first two processes can be disregarded. Under these assumption a mathematical model has been developed describing the vertical concentration gradients in the sediments as a function of external input variation and of certain characteristic parameters (mass sedimentation rate or particle concentration and sinking velocity, distribution factor of the substance between dissolved and particulate phase, hydraulic loading). The model is tested for the distribution of $\mathrm{Pb}, \mathrm{Cd}, \mathrm{Cu}$ and $\mathrm{Zn}$ in Greifensee (Switzerland). Based on sediment data the external input of these metals has increased by the factor $2.5(\mathrm{~Pb}, \mathrm{Cd}, \mathrm{Cu})$ and $4(\mathrm{Zn})$ between the years 1940 and 1975.

Due to the large distribution factors the retention of the lakes lies between $73 \%(\mathrm{Zn})$ and $88 \%(\mathrm{~Pb})$. Despite the variable external input, the sediments are in a quasi-stationary state. The drop in the concentrations of $\mathrm{Cd}, \mathrm{Cu}, \mathrm{Zn}$ in the top $5 \mathrm{~cm}$ of the sediments may indicate that loading has decreased during the last few years. However, this hypothesis must be checked by additional measurements.

\section{RÉSUMÉ}

La reconstruction de l'apport externe dans un lac par l'analyse du sédiment

Le rapport entre la distribution verticale d'une substance dans le sédiment d'un lac et entre le changement temporel de l'apport externe de la substance est compliqué par quatre facteurs: 1. des procès diagénétiques dans le sédiment, 2 . le transport vertical de la substance dans le sédiment (diffusion dans l'eau capillaire, perturbation biologique), 3. variation temporelle de rétention par le lac et 4 . retardement entre l'apport externe et la sédimentation par conséquence du séjour limité d'une substance dans le lac.

Les deux premiers facteurs ne jouent aucun rôle pour les substances conservatrices dans les sédiments, qui montrent des structures stratifiées, si la composante chimique se concentre surtout dans 
la phase solide (par exemple les métaux de trace). Pour ce cas spécial un modèle mathématique est développé, qui décrit l'allure de la concentration verticale dans le sédiment par rapport à une introduction externe variable dans le temps, en fonction de certains paramètres caractéristiques (la sédimentation dans le lac, respectivement la concentration des particules et la vitesse de sédimentation, la profondeur moyenne du lac, la charge hydraulique, le coefficient de distribution de la substance entre la phase adsorbée et dissoute). On teste le modéle dans le lac de Greifensee pour les métaux $\mathrm{Pb}, \mathrm{Cd}, \mathrm{Cu}$ et $\mathrm{Zn}$. Il s'ensuit que l'apport externe de ces métaux a augmenté du facteur $2,5(\mathrm{~Pb}, \mathrm{Cd}$, $\mathrm{Cu})$ à $4(\mathrm{Zn})$ de 1940 à 1975.

A cause du haut coefficient de distribution la rétention d'éléments de trace par le lac sont entre $73 \%$ $(\mathrm{Zn})$ et $88 \%(\mathrm{~Pb})$; les sédiments sont en équilibre avec la charge en dépit de la charge externe variable. Une diminution des taux de métaux dans les $5 \mathrm{~cm}$ supérieurs $(\mathrm{Cd}, \mathrm{Cu}, \mathrm{Zn}$ ) pourrait indiquer une récente baisse de charge, mais cette hypothèse devrait encore être examinée par des recherches ultérieures.

\section{LITERATURVERZEICHNIS}

1 Stumm, W., und Baccini, P.: Man-made chemical perturbation of lakes. In: Lerman, A. (Hrsg.): Lakes-chemistry, geology, physics, p.91-126. Springer, New York 1978.

2 Müller, G., Grimmer, G., und Böhnke, H.: Sedimentary record of heavy metals and polycyclic aromatic hydrocarbons in Lake Constance. Naturwissenschaften 64, 427 (1977).

3 Baccini, P.: Untersuchungen uber den Schwermetallhaushalt in Seen. Schweiz. Z. Hydrol. 38, 121 (1976).

4 Tschopp, J.: Die Verunreinigung der Seen mit Schwermetallen; Modelle fur die Regulierung der Metallkonzentrationen. Diss. ETH Zürich, 1979.

5 Emerson, S.: Early diagenesis in anaerobic lake sediments: chemical equilibrium in interstitial waters. Geochim. cosmochim. Acta 40,925-934 (1976).

6 Emerson, S., und Widmer, G.: Early diagenesis in anaerobic lake sediments - II. Thermodynamic and kinetic factors controlling the formation of iron phosphate. Geochim. cosmochim. Acta 42, 1307-1316 (1978).

7 Imboden, D.M.: Interstitial transport of solutes in non-steady state accumulating and compacting sediments. Earth Planet. Sci. Lett. 27, 221-228 (1975).

8 Guinasso, N.L., Jr., und Schink, D.R.: Quantitative estimates of biological mixing rates in abyssal sediments. J. Geophys. Res. 80, 3032-3043 (1975).

9 Guy, R.D.: The application of a simple chemical model of natural waters to metal fixation in particulate matter. Can. J. Chem. 53, 661 (1975).

10 Imboden, D.M., und Lerman, A.: Chemical models of lakes. In: Lerman, A. (Hrsg.): Lakeschemistry, geology, physics, p. 341-356. Springer, New York 1978.

11 Schindler, P.W.: The regulation of trace metal concentration in natural water system: A chemical approach. In: 1st Speciality Symposium on atmospheric contribution to the chemistry of lake waters. Int. Ass. Great Lakes Res., p. 132-145 (1975).

12 Gaggeler, H., von Gunten, H.R., und Nyffeler, U.: Determination of $210 \mathrm{~Pb}$ in lake sediments and in air samples by direct gramma-ray measurements. Earth Planet. Sci. Lett. 33, 119-121 (1975).

Adresse der Autoren: EAWAG, Úberlandstrasse 133, CH-8600 Dübendorf, Switzerland. 